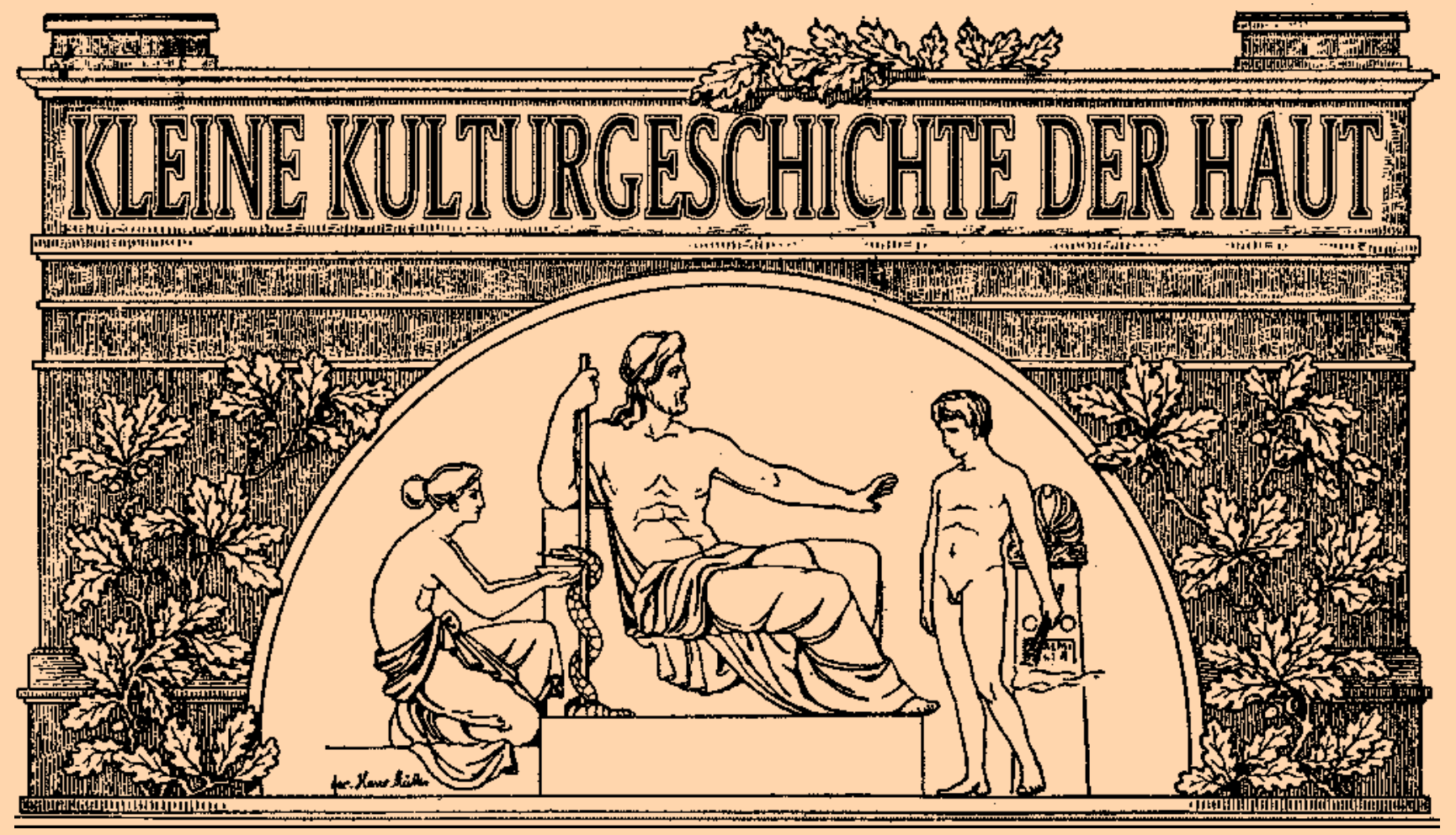

Die Zeitschrift „Aktuelle Dermatologie“ hat mit Beginn des Jahres 2004 diese Rubrik neu geöffnet, um in loser Folge kurze, prägnante und informative Artikel zu publizieren zu vielen Aspekten der Kulturgeschichte in Beziehung zur Haut. Dies erstreckt sich auf alle Kulturen und Zeiten, auf die Künste, auf Mythen und Psychologie und natürlich auch auf medizinische Aspekte.
Unsere Hoffnung, dem geneigten Leser Interessantes $z u$ bieten, den Blick zu weiten und Freude $z u$ bereiten, hat sich auf das Trefflichste bewährt. Reichlich Zusprüche, Kommentare, Vorschläge und aktive Teilnahme haben uns erreicht und erfreut. Dies möge weiter so gehen. Und so werden auch wir fleißig bemüht bleiben, Sie zu erbauen mit unserer kleinen Kulturgeschichte der Haut.

\title{
Literarische Narben: Auf dermatologischer Spurensuche in der Weltliteratur
}

\author{
Literary Scars: Dermatological Traces in Literature
}

Bibliografie

DOI $10.1055 / \mathrm{s}-2006-944876$ Akt Dermatol 2006; 32; 490-495 ๑ Georg Thieme Verlag KG Stuttgart · New York ISSN 0340-2541

Korrespondenzadresse Prof. Dr. Norbert Greiner Institut für Anglistik und Amerikanistik der Universität Hamburg · Von Melle Park 6 . 20146 Hamburg norbert.greiner@unihamburg.de
Im 13. Buch der Odyssee erwacht der Held nach nächtlicher Fahrt auf heimatlichem Boden. Von Athene erfährt er die näheren Umstände der Bedrohung seines Hauses, dem er sich listig in der Gestalt eines Bettlers nähert. Er bleibt für alle unerkannt. Selbst Penelope erkennt in dem „Bettler“, der sie auf die Rückkehr des Odysseus vorbereitet, den Gatten nicht wieder. Nur der Hund Argos spürt hinter der angenommenen Maske die wahre Identität seines alten Herrn auf. Der „Blick“ des Menschen, auch aus dem intimsten Familienkreis, trügt und wird von vorgetäuschter Identität in die Irre geführt. Der Text spitzt damit eine Frage zu, die das Epos in allen seinen Episoden durchgespielt und in den mannigfaltigen Facetten des Problems ausgelotet hat: Die „Suche“ - symbolisch gestaltet in der Irrfahrt - nach den Wurzeln des Ich wirft die entscheidende Frage nach dem Wesen des Selbst und der Anderen, nach dem Ver- und Entbergen von Identität auf. Im gegenwärtigen wissenschaftlichen Jargon lautet die erste Frage: Wie kann sich eine Person in der Gemeinschaft anderer inszenieren, wie kann sie ihrerseits die gesellschaftliche Inszenierung von Wirklichkeit durchschauen und beeinflussen, wie kann man sich in der Welt „verorten“.

\section{Der Bezeichnete: Die Narbe des Odysseus \\ $\nabla$}

Auf dem Höhepunkt des Spannungsbogens, kurz vor der entscheidenden Auseinandersetzung mit den Freiern, ist indes ein erstaunliches retardierendes Moment der Handlungsführung eingeschoben (19. Gesang). Seine alte Amme, die 


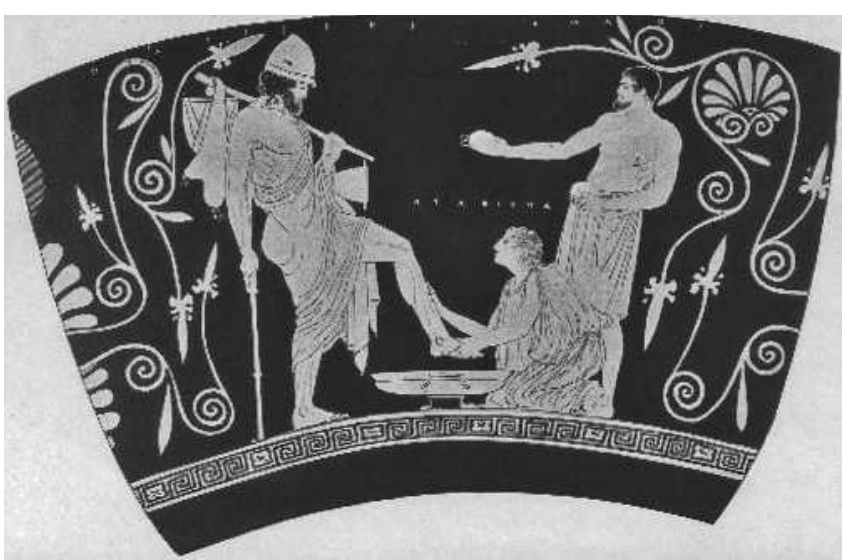

Abb. 1 Die Magd Erykleia erkennt Odysseus an seiner Narbe an der Wade. Attischer Skyphos, ca. 450 v. Chr.

Magd Eurykleia, erkennt, während sie ihm die Füße wäscht, Odysseus wieder. Sie ertastet die Narbe, die ihm in seiner Jugend während einer Jagd von einem Eber zugefügt wurde ( $\bullet$ Abb. 1). Diese [Narbe] nun fand beim Berühren die Alte; sie hatte die Hände Auf und ab beim Waschen bewegt. Da stieß sie den Fu $\beta$ weg, Daß ohne Stütze die Wade ins eherne Becken zurückfiel. Dröhnend kippte das Becken - das Wasser rann auf den Boden. Jubel und Leid miteinander bestürmten ihr Innres, die Augen Gingen ihr über von Tränen, die frische Stimme versagte. Doch sie berührte Odysseus am Kinn und sagte: „Odysseus! Ja du bist es, geliebtes Kind, und - ach - ich begriff es Dann erst, als meinen Herrscher ich ganz und leiblich berührte. [1] Das ist schon ein bemerkenswerter Vorgang. Die Gattin, die ihren Ehemann seit vielen Jahren vermisst, erkennt diesen nicht wieder und lässt sich von seinen Täuschungsmanövern leicht beirren. Die Amme ertastet die Wahrheit - nicht am Aussehen erkennt sie ihn, nicht am Habitus oder an der Stimme, auch nicht an den Augen, sondern an der Narbe am Schenkel. Die Frage nach der Identität des Anderen wird über den Körper verhandelt. Die Person präsentiert sich den Anderen im Rahmen einer Selbstinszenierung als die persona, als die er sich verstanden wissen will. Das Wesentliche entbirgt sich nur in der unmittelbaren, der intimen Begegnung. Selbst das Gespräch mit der Gattin vermag diese Bedingung sozialer Interaktion nicht zu hintergehen. Auch die Amme, solange sie den Bettler lediglich auf dessen äußere Ähnlichkeit mit Odysseus anspricht, kann noch getäuscht werden. Die Narbe aber ist der unausweichliche Ausweis seiner Identität. Deren Ertasten versetzt sie in freudig-erschrecktes Wiedererkennen, nur mit schmeichelnd-drohenden Worten kann Odysseus sie davon abhalten, seine Identität preiszugeben. Doch damit nicht genug. An eben diesem Punkt wird die Erzählung durch einen langen Einschub von etwa 70 Versen unterbrochen, in denen die Entstehung der Narbe geschildert wird: Odysseus weilte als Jüngling bei seinem Großvater Autolykus, von dem der Leser ausführlich berichtet bekommt; dort rüstet er nach Empfang, Gastmahl und Schlaf zur Jagd, die ebenfalls in allen Einzelheiten geschildert wird, ebenso wie die Entstehung der Wunde, das Verbinden, ihre Heilung, die nacheilende Sorge der Eltern. Wozu dient dieser ausgreifende, sich scheinbar im Detail verlierende Exkurs? Erich Auerbach, der unsere Aufmerksamkeit auf diese Stelle gelenkt hat, sieht darin einen Beleg für das homerische Anliegen, die Verhältnisse des wirklichen Lebens in seiner alltäglichen Detailfülle zur sinnlichen Anschauung zu bringen [2]. Ob derartige Intentionen tatsächlich ausgerechnet solch lange Retrospektiven rechtfertigen, sei dahingestellt. In unserem Zusammenhang interessiert uns die weitsichtige Darstellung jener die abendländische Literatur leitmotivisch durchziehenden Frage nach dem Wesen des Selbst und seiner Identitätserfahrung. Und in dieser Hinsicht leistet der Einschub über die Entstehung der Narbe etwas ganz Entscheidendes. Die Narbe erzählt eine Geschichte, die von der Amme in Worte gefasst wird. Der Erzähler sieht sich veranlasst, den Leser in aller Ausführlichkeit damit vertraut zu machen. Die Geschichte der Narbe ist die Biographie des Odysseus; sie beschreibt wesentliche Stadien seines Reifens, denn bei näherer Betrachtung ist das Ereignis ja nichts weniger als ein Initiationsritual. Damit aber ist der scheinbar abschweifende Einschub des Erzählers eng gekoppelt an die Frage der Identität nicht nur in einem identifikatorischen, sondern in einem essentiellen Sinn: Der Ausweis der Einzigartigkeit der Person durch die Narbe wird zugleich zu einem Zeichen ihres Wesens. Die Narbe erzählt die Geschichte von Odysseus als gewordener Person. Diese Geschichte mitsamt ihrem Zeichen erhellt auf eine grundsätzliche Art die Identitätsproblematik. Personale Identität kann dann als gelungen gelten, wenn zwei Bedingungen erfüllt sind: Erstens müssen die Rollenzuschreibungen durch andere weitgehend übereinstimmen mit den Rollenerwartungen, die eine Person für sich selbst entwirft, d.h. die eigene Vorstellung vom Selbst sollte nicht konfligieren mit den Rollenzuschreibungen durch das soziale Umfeld; und zweitens muss eine historische Konstanz in der Biographie vorhanden sein - die Person muss für sich selbst erkennbar in Beziehung stehen zu einer Entwicklung, die sie genommen hat. Das Verhältnis von Person zur sozialen Außenwelt und das Verhältnis zur eigenen Biographie sind die beiden wirkmächtigen Konstituenten einer gelungenen Identität $[3,4]$. Zur Identität gehört nicht nur die Unverwechselbarkeit der Physiognomie, sondern der sinnhafte Zusammenhang einer Lebensgeschichte. Es ist also gewiss kein Zufall, wenn der Erzähler in dem Augenblick, in dem die Amme Odysseus an seinem unverwechselbaren Merkmal identifiziert, sogleich die dazugehörige Geschichte mitsamt einer umfassenden Lebensgeschichte, die immerhin von Initiationsriten handelt, einschiebt. Die Narbe als Körperzeichen ist mehr als ein Ikon, sie erzählt vielmehr eine Geschichte, die den Träger der Narbe über das unverwechselbare Zeichen hinaus mit einer Biographie, einem unverwechselbaren Wesen, ausstattet.

\section{Die Gezeichneten: Brand- und Schandmale romantischer und biirgerlicher Existenzen} $\nabla$

Die Narbe des Odysseus gibt gewissermaßen das Ausgangssignal. Durchgehend spielen derartige oder ähnliche Körperzeichen in der Literatur eine große Rolle, immer sind sie gebunden an identifizierende Funktionen. Die Narbe gewinnt den Charakter einer Hieroglyphe, die den Körper in den allgemeinen semiotischen Prozess des Bezeichnens und Verstehens der Welt als Zeichen und Kommunikationsmedium einbindet.

Vor allem die Epen und Romanzen des Mittelalters und der Folgezeiten, und von dort ausgehend die zunehmende Unterhaltungsliteratur eines breiteren bürgerlichen Lesepublikums, führen dieses Körperzeichen als Standardmotiv für Wiedererkennung oder Identifizierung. Ob es Tristans kaum verheilte Wunde aus dem Kampf gegen Morold ist (die im Übrigen durch eine Kerbe in seinem Schwert gedoppelt wird) oder die Narben manch 
eines ausgesetzten Findelkindes: Auf dem Höhepunkt des Spannungsbogens erzählt die Narbe die entscheidende Geschichte von deren Identität.

Das Phänomen bleibt allerdings weitgehend auf die Unterhaltungsliteratur beschränkt. In den eminenten Werken der Neuzeit tritt die Narbe, so scheint es zumindest, zunächst zurück. Man könnte darin den Ausdruck jenes voranschreitenden Zivilisationsprozesses sehen, den Norbert Elias als einen Prozess zunehmender Affektkontrolle beschrieben hat, die sich nicht zuletzt in der Zurückdrängung der Körperlichkeit äußert [5]. Der Körper verliert in der Aufmerksamkeit der Gesellschaft seine Materialität und damit seine Natürlichkeit. Mit dem Verlust der Unmittelbarkeit verliert der Körper auch seine unmittelbare Zeichenkraft. Mittelbar indessen gewinnt das Zeichen an Bedeutung und wird zugleich komplexer und ambivalenter. Odysseus kann gewissermaßen gar nicht anders, als von der Amme sein Zeichen „lesen“ zu lassen: Der - waschende - Umgang mit dem Körper des Anderen ist so natürlich, dass das Zeichen gar nicht verborgen werden kann (etwa durch die Weigerung, sich waschen zu lassen). In der Neuzeit aber ist das Zeichen am bedeckten Körper grundsätzlich verborgen, seine Präsentation also immer kalkuliert und daher von erhöhter Signifikanz. Die Haltung des Körpers und dessen Gebärden, der Gesichtsausdruck, die Wahl und Tragweise der Kleidung und anderer Schmuckteile werden zum Ausdruck des inneren Menschen, oder genauer: dessen, was er von sich preisgeben möchte. Wir wissen, dass sich mit der zunehmenden Verbürgerlichung des Alltags eine äußere Gleichschaltung der allgemeinen Lebensformen eingestellt hat, die ein Bedürfnis nach Individualisierung hervorrief, welche sich im Verlauf des 19. Jahrhunderts in der immer größeren Vielfalt der Vornamen niederschlug [6]. Eine weitere Möglichkeit der Unterscheidung bestand in der individuellen Zurichtung des Bekleidungsdetails. Die individuell geknüpfte Krawatte, der Schnitt eines Kleidungsstücks, augenfällige Accessoires werden zum Zeichen der individuellen Identität. In diesem neuen weitgehend bürgerlichen - Zusammenhang erhalten auch die Narben einen anderen semiotischen Stellenwert.

Honoré de Balzac entwirft im 19. Jahrhundert das vielleicht umfassendste Sittenpanorama seiner Zeit. Sein mit La comédie humaine betiteltes erzählerisches Gesamtwerk umfasst nahezu alle Berufsstände, soziale Gruppen und soziale Rollen. Die Realgeschichte Frankreichs zur Zeit des Empire, der Restauration und der Julimonarchie bietet den Hintergrund für ein lebendiges Sittenpanorama. In dem Wechselspiel von Umwelt und Charakter auf der Bühne des gesellschaftlichen Lebens gewinnt das Körperzeichen eine besondere Bedeutung. Balzac entwickelt dafür eine eigene Theorie, die er in verschiedenen Essays unter dem Sammeltitel Pathologie de la vie sociale zusammenfasste. Der zunehmenden Gleichschaltung der Individuen im bürgerlichen Stadtleben könne der Einzelne, so Balzacs Überzeugung, nur dadurch begegnen, dass er sich in den Details seiner korporealen Präsentation von den Anderen abhebe - in Kleidung, Haltung, Auftreten. Insofern schenkt er diesen „Zeichen“ des Körpers auch erzähltechnisch besondere Aufmerksamkeit. Ein besonders aussagekräftiges, weil dem Körper durch ein Ereignis eingeschriebenes, Zeichen bleibt jedoch die Narbe, welche, wenn sie zeichenhaft eingesetzt ist, ein besonderes Gewicht bekommt.

Wiederholt führt Balzac seinen Lesern vor, wie der Körper erst gezeichnet wird, um dann die Verhältnisse, in die er eingebettet ist, zu bezeichnen. Der Körper wird zum Teil des Erzählens, zum Bedeutungsträger weit über eine Identitätszuschreibung hinaus. Wir müssen uns mit einem Beispiel begnügen.
Im Mittelpunkt von Glanz und Elend der Kurtisanen steht der Gewaltverbrecher Collin, der sich von einer dämonischen Sträflingsgestalt hinter der Maske eines spanischen Abbés zu einem genialen Menschen mit wilder Energie wandelt, der in ständiger Revolte gegen eine mittelmäßige und korrumpierte Gesellschaft sich schließlich in den Dienst des Staates stellt und das Amt des von ihm überführten Chefs der Sicherheitspolizei übernimmt. Die Wandlung als Höhepunkt der inneren Entwicklung des Protagonisten setzt ein, wenn er und der von ihm lebenslang protegierte Künstler Julien eingekerkert werden. Collin bricht zusammen, wenn er vom Selbstmord des von ihm geliebten Künstlers erfährt, wird aber durch dessen Beispiel (dieser zieht mit dem Selbstmord die Konsequenzen aus seinem Handeln) geläutert und bekehrt. Während der weiteren Gefangenschaft in der Conciergerie versucht der Untersuchungsrichter Camusot zunächst vergeblich, die vermutete Identität des als spanischer Abbé auftretenden Collin zu belegen. Wäre der Abbé, so sein Kalkül, in Wahrheit der ehemalige Strafgefangene Collin, so müsste dieser auf der Schulter ein Brandmal mit den Buchstaben TF (für Travaux Forcés, „Zwangsarbeit“) tragen. Sollte er „das damals durch das Gesetz für bagno-Sträflinge vorgeschriebene Brandmal erhalten haben, würden die Buchstaben beim Beklopfen der Schulter sofort sichtbar werden." [7]. Es kommt schließlich zur Überprüfung der Identität, wie der Untersuchungsrichter diesen Vorgang ausdrücklich bezeichnet.

Der Gerichtsdiener kehrte mit dem Ebenholzstäbchen zurück, das seit undenklichen Zeiten das Kennzeichen ihres Amtes ist und ,Rute' genannt wird. Er schlug damit mehrmals auf die Stelle, auf der der Henker die verhängnisvollen Buchstaben eingebrannt hatte. Darauf kamen in der Haut siebzehn ungleich verteilte Narbenlöcher zum Vorschein. Doch trotz der Sorgfalt, mit der der Rücken untersucht wurde, ließen sich keine Buchstaben erkennen. [...] Carlos bat, man möge das gleiche Verfahren auf der anderen Schulter und der Mitte des Rückens wiederholen. Wie der Arzt auf Wunsch des Spaniers feststellte, zeigten sich etwa fünfzehn weitere Narbenlöcher. Er erklärte, der Rücken sei derart mit Narben übersät, dass das Brandmal des Henkers, sollte es tatsächlich eingegraben worden sein, nicht mehr zum Vorschein kommen könne (S. 306-307). Damit nicht genug. Auch der Chef der Sicherheitspolizei, ein ehemaliger Verbrecher und Bekannter Collins, soll die Identität des Abbé aufdecken. Er kann zunächst „das von Blatternnarben entstellte Gesicht" nicht wieder erkennen; schließlich verweist er auf ein scheinbar untrügliches Identitätsmerkmal: eine „Messerstichnarbe am linken Arm“. Abermals muss Collin seinen Rock ausziehen, und abermals ist diese Narbe verdeckt durch weitere Narben. Beide Narben, die Stichnarbe am Arm und das Brandmal an der Schulter, hatte Collin mit neuen Narben überdeckt, die er sich durch Schusswunden selbst zugefügt hatte. Der Vorgang bietet zwei verschiedene, aber zusammengehörende Deutungsmöglichkeiten. Die Narbe als untrügliches Körperzeichen der Identität wird als Zeichen unlesbar gemacht, indem es durch andere, gleiche Körperzeichen „überschrieben“ wird. Der intelligente Schwerverbrecher weiß um die Aussagekraft des Körperzeichens, er weiß aber auch, dieses Zeichen durch ein noch komplexeres Zeichensystem unlesbar zu machen. Aus dem Körperzeichen als Identitätsausweis wird das Körperzeichen als Identitätsverschleierung. In der zweiten Deutungsmöglichkeit dieses Vorgangs könnte man indes eine Art Vorausdeutung auf den Schluss des Romans erblicken. Der Verbrecher, der angesichts des erlebten Leids eine grundlegende Metamorphose erfährt, löscht nicht nur materiell, sondern in Vorbereitung auf diese Metamorphose auch symbolisch seine alte Identität aus 


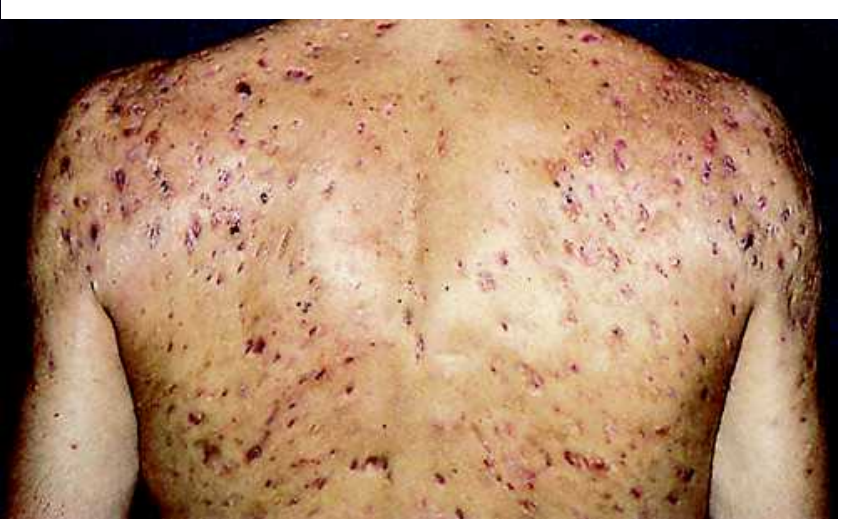

Abb. 2 Flächenförmige Narben verdecken die normale Körperstruktur und deren Charakteristik, ob absichtlich zugeführt oder durch Krankheit (hier Akne conglobata)

und schreibt sich in seinen Körper eine neue, eine vom Leid gezeichnete Identität ein. Wir wissen aus Vater Goriot, dass Collin tatsächlich derartige Brandmale trug. Dort tauchen die Narben auch wieder auf, wenn er auf die Schulter geschlagen wird. Hier nun könnten jene Wunden, mit denen er la marque zu überdecken versuchte, ein Zeichen von Tod und Wiedergeburt sein ( $\bullet$ Abb. 2). Die Auslöschung der alten Narbe und die Neubildung der neuen Narben würden auf diese Weise die Voraussetzung für Collins endgültige Transformation darstellen, die am Ende des Romans mehr ist als eine Maskierung, nämlich eine grundlegende Veränderung seines Selbst. Das Zeichen der Identität wird nun zum Zeichen der spirituellen Integrität und symbolisch gegen die sozial scheinbar Integren, gegen die Nicht-Gezeichneten, ausgespielt. Die Narbe, beziehungsweise die Vielzahl der Narben werden hier zur Metapher für eine innere Identität, die als Körpertext lesbar werden kann und die den identifikatorischen Höhepunkt des Romans darstellt.

Die Verkomplizierung des Körperzeichens spiegelt die Verkomplizierung jener gesellschaftlichen Prozesse, innerhalb derer sich Identitätszuweisungen abspielen, Identitätsfindung und Identifizierung des Gegenübers hinterfragt und problematisiert werden. Ist bei Homer die Narbe des Odysseus Kennzeichen einer sich ihrer selbst vergewissernden Humanität, so sind die Narbenfelder Balzacs möglicherweise Anzeichen einer sich nicht mehr ihrer selbst gewissen Humanität. Die Ausdifferenzierung einer Gesellschaft und ihre freiwillige Unterwerfung unter die ökonomischen Zwänge - bei Balzac ein immer wieder aufgegriffenes Thema - kann an die Stelle von klaren und lesbaren Identitäten offenbar nur noch Verstellungen, Rollen und verworrene Identitäten setzen, denen zwar immer noch Läuterung und Umkehr möglich ist; aber diese Lösungen tragen einen unverkennbaren melodramatischen Beigeschmack. Die Narbe, ehemals Ausweis für das an individuelle Identität gebundene Menschliche, wird zum Zeichen für das Unmenschliche, für die Preisgabe des Menschlichen.

Nirgends wird dies deutlicher als in dem heutzutage durch zahlreiche Literaturverfilmungen ebenfalls einem weiteren Lesepublikum bekannten Roman Frankenstein, or, the Modern Prometheus von Mary Shelley. So wie Odysseus' verborgene Narbe dessen positive Identität bezeichnete, kennzeichnen die vielen Narben des von Frankenstein hervorgebrachten Monstrums eine andere Form von Identität, nämlich den Menschen als Produkt nicht eines göttlichen Schöpfungsaktes, sondern einer hybriden Wissenschaft. Der moderne, sich seiner ethischen Grenzen nicht mehr vergewissernde Wissenschaftler flickt aus alten Knochen ein riesiges Skelett zusammen und verleiht ihm Leben. Als vielnarbiges Monstrum bleibt diesem jedwede humane Identität versagt: Auf der Suche nach Liebe erregen seine Narben und seine Monstrosität nur Abscheu, der künstliche Mensch, das Abbild einer missratenen, weil menschlichen Schöpfung, mutiert zum Dämonen. Wo die eine Narbe als biographisch-historisch gewordenes Zeichen die Identität ihres Trägers auswies, sind nun die vielen Narben Zeichen eines misslungenen, aus dem Geist des Maßlosen hervorgebrachten Versuches, die Schöpfung einzuholen und Leben und Identität zu verleihen. Der Versuch Homers, auf der Suche nach der Identität des Einzelnen das allgemein Humane zu definieren, schlägt bei Shelley um in die Gefährdung des Humanen durch den Einzelnen, der die Grenzen des Humanen glaubt überschreiten zu dürfen. Die zahlreichen Narben erzählen vom künstlichen Schöpfungsprozess des vom Schöpfer Entstellten. Von nun an ist das literarische Bildprogramm in reo Narbe fast ausschließlich negativ besetzt. Es kennzeichnet den physisch Entstellten und oft genug - unter Verwendung vormoderner allegorischer Assoziationsketten - den psychisch und moralisch Entstellten. Das Entstellen wird wörtlich genommen: Der durch die Narben Entstellte hat seine Stelle in der menschlichen Gemeinschaft verloren, ist entstellt.

In der weiteren Literatur des 19. und 20. Jahrhunderts erzählen die Narben der Söldner von dem Leid, das sie erfahren haben, und dem Leid, das sie anderen gebracht haben. Die Narbengesichter der Schurken und Verbrecher machen deren moralischsoziale Entstellung sichtbar, weisen Identitäten aus, die als Kehrseite zum Zivilisationsprozess gehören. Al Capone, die Verbrechergestalt schlechthin, wurde durch Howard Hawks in einem der bekanntesten Filme des 20. Jahrhunderts unter dem Namen Scarface verewigt.

Die Narben des von Frankenstein geschaffenen Monstrums und die Narben der - aus welchen Gründen auch immer - Gebrandmarkten in den Welten Balzacs kulminieren schließlich in Franz Kafkas bedrückender Erzählung In der Strafkolonie. Darin wird ein Forschungsreisender, der die Strafkolonie einer europäischen Großmacht besucht, Zeuge einer Todesstrafe, die mit einem „eigentümlichen Apparat“ ausgeführt wird: dem zu Tode Verurteilten, der auf diesen Apparat gefesselt ist, wird mit Hilfe eines komplizierten Mechanismus das übertretene Gebot und die Schuld in den Leib eingestanzt. Seine Schuld ist „zweifellos“, verteidigen kann er sich nicht, das Urteil wird gnadenlos ausgeführt. Im Verlauf der Exekution kann der Verurteilte seinen zunehmend vernarbten Körper entziffern. Er erfährt, als wen man ihn begreift, und diese letzte Identitätszuschreibung ist identisch mit dem Vollzug der Hinrichtung, mit der Auslöschung von Identität.

Wenn aufgrund des Einspruchs, den der Besucher der Strafkolonie gegen die „Ungerechtigkeit des Verfahrens und die Unmenschlichkeit der Exekution“ [8] erhebt, sich der ausführende Offizier auf das Straflager begibt und der Maschine anordnet, sie solle ihm das Gebot „Sei gerecht! “ einschreiben, zerstört sie sich selbst und mit ihrer Selbstzerstörung auch den an sie gebundenen Offizier. Die in dieser Erzählung aufgeworfene kafkaeske Frage der Daseinsschuld und der vergeblichen Rebellion gegen undurchschaubare Gesetzesordnungen kann in dem hier zur Verfügung stehenden Raum nicht näher verfolgt werden. Es mag uns aber als Beispiel für die Versuche auch der modernen Literatur dienen, Erkenntnisprozesse - wie hier in der Form des Wiedererkennens oder der Einsicht in tatsächliche oder vermeintliche Schuldverstrickungen - in Form von Textnarben in 
Körper einzuschreiben. Aus der Komplexität und Ambivalenz der Zeichennarben bei Balzac ist die (fast) endgültige Unlesbarkeit des Narbenzeichens in der Moderne geworden - als Kennzeichnung einer Welt, die sich vollends der Lesbarkeit entzieht, weil der Glauben an die Lesbarkeit der Welt die Existenz von etwas zu Lesendem voraussetzt. Das zwar noch entzifferbare, aber sich der eindeutigen Deutung widersetzende Körperzeichen bezeichnet eine im Grundsatz verrätselte Welt, die ohne Offenbarung und daher Lesbarkeit auszukommen hat.

\section{Der Aus-Gezeichnete: Ahabs Kampf gegen das Leben \\ $\nabla$}

Eine vermittelnde Stellung zwischen der mythisch-heroischen Überhöhung des Odysseus in der Antike, der Bezeichnung komplexer bürgerlich-alltäglicher Lebensformen im Realismus des 19. Jahrhunderts und der epistemologischen Verrätselung der Welt durch den Modernismus nimmt in mancherlei Hinsicht eines der monumentalsten Romanwerke der Weltliteratur ein: Herman Melvilles Moby-Dick.

„Nennt mich Ismael“, lädt der Ich-Erzähler den Leser eingangs ein und stilisiert sich damit zu einer erzählenden persona, die sich analog zu seinem alttestamentarischen Prototyp aus dem heilsgeschichtlichen Versprechen des auserwählten Volkes Israel ausgeschlossen sieht: Nur geht die melancholische Weltentsagung von ihm selbst aus, weil er einer dem ökonomischen Geist geschuldeten Enge und Korrumpiertheit entfliehen möchte und auf der Weite der Weltmeere die Freiheit der Kontemplation sucht. Aus der Perspektive des melancholischen Zivilisationskritikers Ismael begegnen dem Leser die eigentlichen Antagonisten des Romans, der Weiße Wal und dessen Jäger, Kapitän Ahab. In geschickter Spannungsführung und im übrigen eingebettet in eine Fülle des realistischen Details, die an Homer und an Balzac erinnert (die Techniken des Walfangs und die Realien der Walfangindustrie sind in langen Passagen geschildert), wird ausführlich vom Vorhaben, der beginnenden Fahrt und dem in seiner Kajüte eingeschlossen bleibenden Kapitän erzählt, bevor dieser schließlich, im 28. Kapitel, auf der Szene erscheint:

[...] als ich den Blick nach der Heckreling hob, überlief mich ein ahnungsvoller Schauder. Doch mein banges Vorgefühl verblasste vor der Wirklichkeit: auf dem Achterdeck stand Kapitän Ahab! [...] Er sah aus, als hätte man ihn vom Scheiterhaufen herabgerissen, nachdem der Feuersturm den ganzen Leib verheert, doch nicht verzehrt und seine von den Jahren gefestigte Kraft nicht um eines Haares Breite geschmälert hat. Seine hohe, breitschultrige Gestalt war wie aus schwerer Bronze und in unwandelbarer Form gebildet, Cellinis erzenem Perseus gleich. Aus seinem grauen Haar hervor und auf der einen Seite des lohbraun versengten Angesichts und Halses steil nach unten, bis es im Rock verschwand, drang weißlich, leichenfahl anzusehen, ein gertendünnes Mal. Es glich der Wunde an dem stolz aufragenden Stamm eines hohen Baumes, wenn der Blitz zerfetzend niederfährt und, ohne einen einzigen Zweig zu knicken, die Rinde senkrecht vom Wipfel bis zur Wurzel aufreißt und höhlt, bevor er in den Boden schlägt: der Baum bleibt noch voll frischen Lebens, doch er ist gezeichnet. Ob dieses Mal ihm angeboren oder die Narbe einer grässlichen Wunde war, das konnte niemand mit Gewissheit sagen. (Kap. 28, 154). [9]

Alles konzentriert sich im Folgenden auf Ahab. Wir sehen eine Narbe, die von einem an sie gebundenen Ereignis erzählt, der Jagd nach dem Weißen Wal, und wir erleben die Deutungsversuche des Erzählers. Durch dessen Interpretationsleistung vermit- telt erschließt sich dem Leser die möglicherweise symbolische Tatsache, dass Ahab den Walfang vierzig Jahre lang betrieben hat (Kap. 82), also über jenen Zeitraum hinweg, den das erwählte Volk Israels nach dem Auszug aus Ägypten in der Wüste umherzog, und er vergleicht das Gelobte Land der Israeliten mit dem kläglichen Ergebnis der lebenslangen manischen Suche Ahabs. Der Leser teilt mit dem Erzähler die Assoziationskette, die das Symbol des Weißen Wals auslöst, und die mehr oder weniger bewussten Reflexionen auf die mythisch-archetypischen Dimensionen der Figur, der Jagd und des Gejagten.

Dass es sich bei der Narbe um mehr handelt als um eine Verletzung im Kampf, ergibt sich aus den Mutmaßungen über ihre Genese, aus der Bindung der Narbe an einen rätselhaften und in seiner Besessenheit zugleich faszinierenden Charakter und wiederum dessen Bindung an, genauer: Fixierung auf den Weißen Wal. Ob es sich beim Wal um ein Symbol sublimierter Sexualität oder um ein traditionelleres religiös-mythisches Bild in der Tradition der Inder oder Hebräer (Jonas und Hiob) handelt, um eine Analogie zu den Drachen des Perseus und des St. Georg bis zu Hobbes' Leviathan oder das Symbol göttlicher Unbeflecktheit und Macht [10-13], bleibt in unserem Zusammenhang unerheblich, solange wir im Blick behalten, dass es sich beim Wal nicht um ein arbiträres Alltagszeichen, sondern um ein universelles Symbol in einer eminenten historischen Tradition handelt. Solange er sich nicht auf eine Bedeutung festlegen und einengen lässt, bietet er eine Projektionsfläche für mancherlei Sinnkonstruktionen, die eine jede Leserschaft aufs Neue in ihn hineinlesen kann. „Moby Dick bedeutet - das lehren die zahlreichen Aussagen über den Wal im Buch selbst wie in der Melville-Forschung - alles und nichts. Er symbolisiert das Böse ebenso wie das Prinzip des Lichts“ ([14], S. 322). Doch selbst einem voraussetzungslosen, naiven Leser erschließt sich die Grundstruktur des Textes: ein bis zur Selbstaufgabe reichender Kampf eines von der Kampfidee besessenen Mannes gegen einen übermächtigen Gegner, den er unter Vernachlässigung aller Rücksichten und ohne Hoffnung auf einen Sieg bis in den eigenen Tod bedingungslos und unter hohen Verlusten verfolgt. Alle konkreten Ausdeutungen lassen sich letztendlich auf die vorwissenschaftliche Formel des Erzählers bringen, mit der er das Meer, dessen Herr der Wal ist, zu umschreiben sucht: „Das nie zu fassende Trugbild des Lebens“ (Kap. 1, 24 ).

Was aber symbolisiert dann Ahabs Narbe? Ahab jagt den Herrscher jenes „nie zu fassenden Trugbilds des Lebens“ mit dem Ziel, ihn zu vernichten. Das aber ist ein dämonisches, selbstmörderisches Unterfangen, das - wie der Roman zeigt - zur Selbstverstümmelung führt. Sollte, wie der Erzähler als Interpretationsmöglichkeit anbietet, die Narbe den ganzen Körper hinunter sich fortsetzen, dann wäre sie auch Zeichen der (symbolischen) Kastration - die im übrigen wiederholt in Bildern angedeutet ist, etwa, wenn der Kapitän sich sein Holzbein in den Leib rammt -, das Schicksal dessen, der sich zum erklärten Feind des Lebens macht. Längst ist deutlich geworden, dass die Narbe Ahabs nicht nur eine korporeale Verstümmelung ist, sondern geistiger Natur: in allen Deutungsmöglichkeiten Ausdruck eines von vornherein paradoxen Versuchs, das Leben zu besiegen. Ahabs fieberhafte Jagd über die Weltmeere erinnert nicht von ungefähr an die „Suchreise“ des Odysseus; er hat die metaphysische Dimension dieses archetypischen Verhaltens komplett verinnerlicht, deren Ziel seit jeher darin liegt, „die Beziehung des Helden zur Gruppe, zum Schicksal und zu sich selbst auszuloten" ([14], S.11). Ahab aber kehrt die Selbstvergewisserung fast in ihr Gegenteil. Er rebelliert gegen das Schicksal, das dem 
Menschen mit dem Leben, das ihm gegeben wird, auferlegt ist, wie immer wir es ausdeuten. Seine „quest“ ist Selbstverleugnung.

Daneben ist die Narbe auch das Zeichen einer psychischen Verstümmelung. Ahab hat sich mit seiner Fixierung auf den Wal dem Leben verweigert. Von der allmählichen Offenbarung seines Wahns bis zu dem Punkt, an dem er einem anderen Kapitän aus seinem Heimathafen die Hilfe bei der Suche nach dessen vermisstem Sohn verweigert, wird deutlich, wie sehr er sich aus der menschlichen Gemeinschaft entfernt und sein eigenes Schiff samt Besatzung zu einer in sich geschlossenen Wahnwelt gemacht hat (Kap. 80). Die Körperzeichnung ist gleichsam das Fanal des outlaws.

Folgen wir der kritischen Vernunft des Erzählers, ist der Weiße Wal das Symbol einer erhabenen Utopie, in der der „Glanz uranfänglicher Zeiten neu belebt“ wird, Ausdruck eines Schöpfungszustands vor dem Sündenfall. Ismael stellt sich der Erhabenheit des Jagdobjekts. Er weiß, dass man das in diesem Tier Verkörperte niemals überwältigen und schon gar nicht beherrschen kann. Er ist der einzige, der überlebt. Wer wie Ahab „das Leben“ besiegen möchte, geht zugrunde. In der Narbe Ahabs und seiner sonstigen körperlichen Versehrtheit ist dieses Schicksal präfiguriert: denn sie resultiert realiter aus einem vorausgegangenen Kampf mit dem Wal. Das Körperzeichen Ahabs entsprang seiner körperlichen Auseinandersetzung mit dem Wal und bezeichnet seinen monomanen Bezug auf ihn:

[...] als seine Niederlage ihn dann zur Heimkehr zwang, als Ahab und seine Pein Tage, Wochen, Monate zusammengeschmiedet in der Hängematte hingestreckt lagen, als er mittwinters das düstere, sturmumheulte Kap an Patagoniens Küste umsegelte - da geschah es, daß sein zerstörter Leib und seine klaffende Seele blutend ineinanderströmten, sich vermengten; und das verwirte seinen Geist. (Kap. 41, 221).

Worin aber liegt die Faszinationskraft, die von dieser Figur ausgeht? Melville verstand seinen Roman, den C. G. Jung im Übrigen für „den größten amerikanischen Roman“ [15] hielt, als einen Beitrag zu einer sich vom englischen Vorbild absetzenden amerikanischen Nationalliteratur. Diese programmatische Absicht erforderte unter anderem eine neue, der feudalistisch-hierarchischen Gesellschaftsordnung Englands entgegenwirkende Konzeption des tragischen Helden. In dem vom Leben versehrten, sich dem Leben hasserfüllt entgegenstemmenden Individuum, das im Wissen um seinen Wahn diesen bis zum eigenen Untergang weiterverfolgt, entwickelt Melville eine Struktur des unentrinnbaren Widerspruchs, der der Struktur der tragischen Begebenheit nicht unähnlich, aber in seinen Augen ungleich moderner ist.

\section{Literarische Narben im Buch des Lebens \\ $\nabla$}

Das Buch, das dem Menschen zum Lesen gegeben ist, als Himmelsbuch, als Buch der Natur, als Offenbarungsschrift, setzt zwar hermeneutischen Sachverstand voraus, unterstellt aber zugleich prinzipielle Lesbarkeit. Das Zeichen und dessen Entzifferung werden zur kulturellen Grunderfahrung des Menschen. In diesem semiotischen Prozess nimmt die Erfahrbarkeit individueller Identität, des Selbst und des Anderen, eine besondere Stellung ein; sie äußert sich in kulturellen Bezeichnungen (Namen, Kennzeichnung der Zugehörigkeit) und in Körperzeichen („besondere Merkmale“). Auch in der Welt Homers gelten die Welt und der in ihr behauste Mensch als lesbar. Diese prinzipielle Da- seinsentzifferung offenbart sich nicht zuletzt in der unmittelbaren Korporealität des Identitätsausweises, an der Narbe, die ein unverwechselbares, nicht korrigierbares Zeichen von individueller Identität ist. Allerdings weiß Homer um die trügerische Kraft kultureller Zeichen - Habitus, Kleidung und Rede dienen oft genug der Verstellung, Verführung oder Verwechslung, während der Rückgriff auf das unhintergehbare Körperzeichen zu deren Entdeckung führt.

Der Zivilisationsprozess wäre auch zu schreiben als ein Prozess des Verlustes von Lesbarkeit: Nicht nur gestaltet sich in der Neuzeit die Selbsterfahrung problematisch, auch die Selbstverrätselung des Individuums als Teil seiner sozialen und kulturellen Selbstinszenierung gewinnt an Bedeutung. „Nicht mehr die Gottheit verbirgt sich vor ihren Geschöpfen in der Natur, sondern diese verbergen sich voreinander in ihrer Kultur“ [16]. Die Meisterschaft der Entzifferung beherrscht Jahrhunderte lang die kulturellen Diskurse. Das der unmittelbaren Wahrnehmung zugängliche Körperzeichen verliert zwar seine Eindeutigkeit, bietet sich aber immer noch als ikonisches Refugium aus der ausdifferenzierten Welt des Kulturmenschen dar, der in allen seinen Facetten begriffen wird. Die Narbe als Verweis auf den „neuen“ tragischen Helden der ebenfalls „Neuen“ Welt vermag schließlich noch den Helden als solchen zu bezeichnen, doch bleibt dessen Ausdeutung, philosophisch und moralisch, nach allen Seiten hin offen - so als würde die „Spaltung“ der Person Ahabs durch die Narbe auch die grundsätzliche Ambivalenz eines jeden Zeichens symbolisieren. Die Narbe ist das einfachste und zugleich komplizierteste Zeichen zur Bezeichnung menschlicher Identität.

\section{Literatur}

1 Homer. Odyssee. Griechisch und Deutsch. Übertr. von Anton Weiher. 11. Auflage. Düsseldorf: Artemis \& Winkler, 2000

2 Auerbach E. Mimesis. Dargestellte Wirklichkeit in der abendländischen Literatur (1946). Bern: Francke, 1988: 8

3 Mead GH. Mind, Self, and Society: From the Standpoint of a Social Behaviorist (Hrsg. Morris CW). Chicago: University of Chicago Press, 1970

4 Erikson EH. Identity and the Life Cycle. New York: Norton \& Co, 1994

5 Elias N. Über den Prozeß der Zivilisation. Soziogenetische und psychogenetische Untersuchungen, Band I: Wandlungen des Verhaltens in den weltlichen Oberschichten des Abendlandes. Frankfurt/M: Suhrkamp, 1997: 198

6 Corbin A. Histoire de la vie privée (Hrsg. Ariès P, Duby G). Bd. 4: De la Révolution à la Grande Guerre (Hrsg. Perrot M). Paris: Editions du Seuil, 1987: 419-436

7 de Balzac H. Glanz und Elend der Kurtisanen. Wiesbaden: Vollmer, o. J: 302

8 Kafka F. In der Strafkolonie. In: Das Urteil und andere Erzählungen (1935). Frankfurt/M: Fischer, 1952: 110

9 Melville H. Moby Dick. (Übers. A. u. H. Seiffert). Berlin: Aufbau Taschenbuch Verlag, 2005

10 Staats A. Melville. Moby-Dick. In: Lang HJ (Hrsg). Der amerikanische Roman. Düsseldorf: Bagel, 1972: 103-141

11 Olson C. Call Me Ishmael (1947). New York: Grove Press, 1958

12 Hoffman D. Myth, Magic, and Metaphor in Moby-Dick. In: Form and Fable in American Fiction. (1961). New York: Norton \& Co, 1965

13 Brumm $U$. Die religiöse Typologie im amerikanischen Denken. Leiden: Brill, 1963

14 Schulz D. Suche und Abenteuer. Die „Quest“ in der englischen und amerikanischen Erzählkunst der Romantik. Heidelberg: Carl Winter Universitätsverlag, 1981

15 Jung CG. Psychologie und Dichtung. In: Ermatinger E (Hrsg). Philosophie der Literaturwissenschaft. Berlin: Junker und Dünnhaupt, 1930: 316

16 Blumenberg H. Die Lesbarkeit der Welt. 2. Aufl. Frankfurt/M: Suhrkamp, 1983: 111 (hier auch weitere Ausführungen zu diesem Gedanken: $108-120)$ 